\title{
Características del desempeño ocupacional de un grupo de adultos que viven con VIH-SIDA
}

\author{
Angélica María Monsalve Robayo ${ }^{a}$, Martha Rocio Torres Narváez ${ }^{a}$, \\ Edgar Debray Hernandez Alvarez ${ }^{a}$, Angela González Puche $e^{b}$, Maira Lizeth Peña Duarte, \\ María Angélica Zúñiga Peña ${ }^{\mathrm{a}}$ \\ ${ }^{a}$ Universidad del Rosario, Bogotá, Colombia.

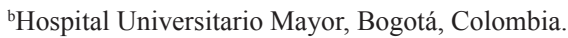

\begin{abstract}
Resumen: Introducción: El virus de inmunodeficiencia humana marca la vida de las personas, principalmente en el autocuidado, el trabajo y el tiempo libre, lo cual influye en el ejercicio de la participación social. Objetivo: describir el desempeño ocupacional de 29 pacientes que viven con VIH SIDA y son atendidos por el programa B24 en un Hospital de Bogotá-Colombia. Metodo: estudio descriptivo-exploratorio, recaba información sobre patrones del desempeño, roles ocupacionales e independencia en actividades instrumentales de la vida diaria (AIVD). Se realiza una entrevista y se utiliza el cuestionario ocupacional, el listado de roles y el índice de Lawton y Brody; se procesan los datos en el programa SPSS y se realiza un análisis de frecuencias. Resultados: los participantes tienen en promedio 53 años, el $82.8 \%$ son hombres y el $48.3 \%$ un nivel de formación superior. Trabajan en promedio 2 horas diarias, invierten en actividades cotidianas alrededor de 11 horas diarias, casi 5 horas a la recreación y 6 horas al descanso. Los roles más significativos son amigo y miembro de familia y el menos significativo miembro de un grupo religioso. El $65.5 \%$ es independiente en AIVD, el 31\% precisa ayuda para algunas actividades y el 3.4\% es dependiente. Los signos y síntomas del VIH y las infecciones oportunistas, dificultan la asunción de roles, incluyendo las actividades socio-laborales. Conclusión: Cobra valor el significado de la transición ocupacional que vive este colectivo y su perspectiva de futuro para implementar programas que propendan por la re-significación de su participación socio-familiar.
\end{abstract}

Palabras-clave: Análisis y Desempeño de Tareas, VIH, Terapia Ocupacional.

\section{Características de desempenho do trabalho de um grupo de pessoas com HIV-AIDS}

Resumo: Introdução: O vírus da imunodeficiência adquirida afeta a vida das pessoas, principalmente no autocuidado, trabalho e lazer, assim como o exercício da participação social. Objetivo: Descrever o desempenho ocupacional de 29 pessoas que vivem com HIV e AIDS e recebem atenção do programa B24 em um hospital em Bogotá-Colômbia. Método: Estudo descritivo e exploratório sobre os padrões de atuação, papéis ocupacionais e independência nas atividades instrumentais da vida diária (AIVD). Foram usados uma entrevista e um questionário ocupacional, o índice de Lawton e Brody. Os dados foram processados no SPSS para a análise de frequência das variáveis. Resultados: Os participantes têm em média 53 anos, 82,8\% homens e 48,3\% com formação em educação superior. Eles trabalham uma média de 2 horas por dia e dispendem 11 horas por dia para as atividades diárias, quase 5 ou 6 horas de lazer e descanso. Os papéis mais significativos são de amigo e membro da família, o menos significativo é ser membro de um grupo religioso. $65,5 \%$ das pessoas são independentes em AIVD, 31\% precisam de ajuda para algumas atividades, só 3,4\% são dependentes. Sinais e sintomas de HIV e infecções oportunistas dificultam que se assumam

Autor para la correspondencia: Angélica María Monsalve Robayo, Universidad del Rosario, Cra 24 No 63c-69, CP 111221, Bogotá, Cundinamarca, Colombia, e-mail: angelica.monsalve@urosario.edu.co.

Recibido Nov. 13, 2015; $1^{\circ}$ Revisión Feb. 18, 2016; $2^{\circ}$ Revisión Marzo 15, 2016; Aceptado Abr. 13, 2016. 
papéis, incluindo atividades de trabalho. Conclusão: o valor Cobra demonstra o significado de transição profissional para este grupo e suas perspectivas futuras para implementar programas que promovam a importância da sua participação social e familiar.

Palavras-chave: Análise e Desempenho de Tarefas, HIV, Terapia Ocupacional.

\title{
Occupational performance characteristics of a group of adults with HIV-AIDS
}

\begin{abstract}
Introduction: The human immunodeficiency virus affects the lives of people especially regarding self-care, work and leisure time, factors that compromise the exercise of social participation. Objective: To describe the occupational performance of 29 patients living with HIV and AIDS assisted by the B24 program in a hospital in Bogotá-Colombia. Method: Descriptive and exploratory study on patterns of performance, occupational roles and independence in instrumental activities of daily living (IADL). An interview was conducted and an occupational questionnaire, the Lawton and Brody index, was used. Data were processed in SPSS and frequency analysis was performed. Results: Participants were 53 years old in average, $82.8 \%$ were men and $48.3 \%$ had higher education. They work an average of 2 hours a day and spend 11 hours a day with daily activities, and almost 5 or 6 hours with leisure and rest. The most significant roles are friend and family member, and the least significant, member of religious groups. Among participants, $65.5 \%$ are independent to develop IADL, 31\% need help for some activities and 3.4\% is dependent. Signs and symptoms of HIV and opportunistic infections make it difficult to assume roles, including work activities. Conclusion: Cobra value demonstrates the meaning of occupational transition for this group and its future perspective to implement programs that may foster the importance of their social and family participation.
\end{abstract}

Keywords: Task Performance and Analysis, HIV, Occupational Therapy.

\section{Introduccion}

El VIH-SIDA se ha tornado en un tema central de salud pública a nivel mundial; ello a causa de las alarmantes cifras de contagio y mortalidad, en parte debido al bajo acceso a un tratamiento oportuno. Según las últimas estimaciones realizadas, en el mundo hay 33.3 millones de personas que padecen el VIH/SIDA y en el 2009 se produjeron 2.6 millones de nuevas infecciones. En la situación epidemiológica actual del VIH/SIDA en el mundo, Latinoamérica ocupa el cuarto lugar en incidencia y prevalencia, seguido de África, Europa y Asia (TEVA et al., 2012).

Colombia ha sido clasificada como un país donde el VIH/SIDA es una epidemia muy concentrada, debido a su rápida propagación por una o más poblaciones aunque no se haya extendido entre la población en general (COLOMBIA..., 2014), se encuentra en la primera posición en porcentaje de hombres jóvenes con VIH/SIDA y en segunda posición en porcentaje de niños (TEVA et al., 2012). Colombia se encuentra en el puesto 34 dentro de la comparación realizada entre 122 países, con una cifra de 136.500 personas infectadas con VIH y con vida al final del año (CENTRAL..., 2014)

El Ministerio de Salud y Protección Social de la República de Colombia (2014), afirma que desde
1985 al 31 de diciembre de 2012 se ha reportado un total de 95.187 casos nuevos de infección por VIH Sida lo que evidencia el incremento anual de casos a nivel nacional. Lo anterior refleja una situación inquietante en el país y la necesidad de implementar proyectos para su prevención y acceso al tratamiento.

En Colombia la ley 972 de 2005, establece los lineamientos para mejorar la atención a personas con enfermedades catastróficas, específicamente el VIH SIDA y ratifica la importancia de considerar el manejo del estigma como un elemento principal (COLOMBIA, 2005). Por otro lado, se ha evidenciado el progreso en cuanto a programas preventivos y acceso al tratamiento, lo cual ha reducido la cantidad de muertes y contagios. El informe del día mundial del SIDA reporta una reducción en un 50\% de la infección por VIH en 25 países de ingresos bajos y medios; cabe anotar que la calidad de vida de las personas está directamente asociada al sentido que tiene ésta y cómo se reconoce la posibilidad de involucrarse con el tratamiento médico y los cambios generados a nivel social.

A pesar del progreso en cuanto a la reducción de la infección y la implementación de proyectos de salud pública, se ha desarrollado y mantenido un estigma que afecta directamente el desempeño ocupacional de la persona portadora de VIH, entendiendo desempeño ocupacional como el producto de la interacción 
entre el sistema humano, la tarea y el ambiente (KIELHOFNER, 1995). Ha sido posible destacar que el estigma presente conlleva a la restricción en la participación de las personas contagiadas por el virus de inmunodeficiencia.

El estigma no solo se contempla a partir del origen del VIH sino, a su vez existe a causa de su rápida expansión y adquisición. Teniendo en cuenta lo anterior, es indispensable promover hábitos de vida saludables que fomenten y aseguren un desempeño ocupacional satisfactorio en las distintas áreas ocupacionales afectadas; las actividades de la vida diaria, el trabajo y/o la escolaridad. Lo anterior deja entrever cómo éstas áreas y en específico la cotidianidad de la persona se ven enfrentadas al apartheid ocupacional, definido como, la

[...] segregación de grupos de personas mediante la restricción o negación de su acceso a una participación digna y significativa en las ocupaciones de la vida diaria [...] (KRONEMBERG; SIMO; POLLARD, 2007, p. 66).

El estigma social, la sintomatología que produce la enfermedad y el tratamiento farmacológico antirretroviral, impactan el desempeño de hábitos y rutinas del individuo, aspectos en los que Terapia Ocupacional puede intervenir, para mejorar las áreas ocupacionales referidas al trabajo, el ocio productivo y la escolaridad en los diferentes contextos (BRAGANÇA; PALHA, 2011).

De acuerdo con la literatura, se afirma que las personas que viven con VIH/SIDA se ven enfrentadas a distintos problemas psicosociales, físicos y cognitivos que limitan el tipo y la cantidad de actividades que ellos quieren y son capaces de realizar (BEDELL, 2000), lo cual impacta el desempeño de roles ocupacionales principalmente aquellos que requieren de la interacción y participación como miembro de la comunidad, de la familia y del equipo de trabajo (KIELHOFNER et al., 2008; GUTTERMAN, 1990).

La intervención desde Terapia Ocupacional muestra resultados significativos que benefician a los pacientes que viven con VIH SIDA ya que ésta promueve la calidad de vida, reduce el sentimiento de soledad y aumenta el bienestar, permitiendo respuestas adaptativas a las demandas del entorno (GUTTERMAN, 1990; BARKEY et al., 2009). La terapia ocupacional en personas que viven con VIH/SIDA focaliza su atención hacia la posibilidad de conservar o asumir funciones de productividad necesarias o deseadas para un mejor desempeño ocupacional y calidad de vida. Este entendido como la transacción que existe entre la persona, la tarea y el ambiente y cómo ello supone un trabajo desde la volición, habituación y capacidad de desempeño (KIELHOFNER, 1995). Las funciones de productividad se componen de rutinas y tareas que permiten a una persona desempeñarse exitosamente en las distintas áreas de ocupación (actividades de la vida diaria, actividades instrumentales de la vida diaria, descanso, sueño, educación, trabajo, juego, ocio/tiempo libre y participación social) las cuales se ven afectadas durante el curso de VIH/SIDA. Por lo cual el presente estudio busca describir el desempeño ocupacional de pacientes q viven con VIH SIDA, de una Institución Prestadora de Servicios de Salud - IPS en Bogotá-Colombia.

\section{Metodo}

Se trata de un estudio de tipo descriptivo-exploratorio. Con un grupo de pacientes que viven con VIH SIDA. Los criterios de inclusión fueron: (1) Pertenecer al programa B24 del Hospital Universitario Barrios Unidos, (2) Ser mayor de 40 años, (3) Saber leer y escribir, (4) Recibir tratamiento con terapia antirretroviral, (5) Realizar marcha independiente y (6) Comprender las tareas a ejecutar. Criterios de exclusión: individuos (1) hospitalizados en los últimos seis meses, (2) con una condición médica no controlada o síndrome de inmovilidad, (3) con deficiencias osteomusculares que afectaran su desempeño en la tarea motora asignada (por ejemplo con diagnóstico de artrosis y con amputaciones). Se convocan inicialmente a 32 participantes en el proyecto, sin embargo dos no cumplían los criterios de inclusión y uno se negó a participar.

\subsection{Consideraciones éticas}

El estudio cuenta con aval del Comité de Ética en Investigación de la Escuela de Medicina y Ciencias de la Salud, de la Universidad del Rosario. Fue realizado de acuerdo con los estándares locales e internacionales de ética en investigación con seres humanos. De acuerdo con la resolución 08430 de 1993, el estudio tiene riesgo mínimo, porque es prospectivo y emplea el registro de datos a través de procedimientos comunes, que incluyen la anamnesis y exámenes físicos de diagnóstico rutinarios, sin realizar una intervención directa con la población (COLOMBIA, 1993). La información se ha usado con fines exclusivamente académicos y se mantiene la privacidad de los participantes con el empleo de códigos, en lugar de sus nombres y apellidos. El estudio cuenta con el aval del uso de los datos, por parte de la institución que atiende a 
los pacientes. Los pacientes autorizaron el acceso a sus datos de la historia clínica. Los investigadores principales custodian la información en la Institución Prestadora de Servicios de Salud en la que se realiza el proyecto, la cual tiene acceso restringido a los investigadores. Se elaboró y se aprobó por parte del Comité de Ética el consentimiento informado, el cual fue diligenciado y firmado por los participantes, dos testigos y el investigador principal.

\subsection{Procedimientos recolección de datos}

El área de trabajo social de la institución (IPS) informó a los pacientes sobre el proyecto, los interesados se inscribieron para recibir mayor información. Fueron reclutados vía telefónica con mayor información del proyecto y a los interesados se les asignó cita. A partir de un formato desarrollado por el grupo investigador se recolectaron datos de historia clínica, datos ocupacionales con la aplicación de instrumentos seleccionados: cuestionario ocupacional diseñado para conocer los patrones de desempeño y la percepción y motivación que tiene el usuario frente a los mismos (RIOPEL; KIELHOFNER; HAWKINGS, 1986); el listado de roles ocupacionales adaptado para identificar el rol más y menos significativo para cada persona y conocer los cambios generados en el transcurso del tiempo, (OAKLEY; KIELHOFNER; BARRIS, 1986); y el índice de Lawton y Brody, para establecer el nivel de independencia a la hora de realizar sus actividades instrumentales de la vida diaria (JIMÉNEZ et al., 2012). A los pacientes les fueron aplicadas las tres pruebas seleccionadas por el grupo investigador. La información se registró en un formato de entrevista inicial programado en tablas dinámicas elaboradas en Excel.

\subsection{Procesamiento y análisis de datos}

A los datos registrados les fueron aplicadas las pruebas de normalidad de Kolmogorov Smirnov (NEHER; LEITNER, 2010), se realizaron los análisis de frecuencias de cada una de las variables con sus medidas de dispersión utilizando estadísticas descriptivas a través del programa SPSS versión 20.

\section{Resultados}

El estudio se realizó en el periodo comprendido entre Agosto del 2013 a Mayo del 2014 la inclusión de los pacientes aparece en la Figura 1 - Flujograma. El promedio de edad de la población evaluada fue 53 años (DS +/- 7.51), 82.8\% de sexo masculino y

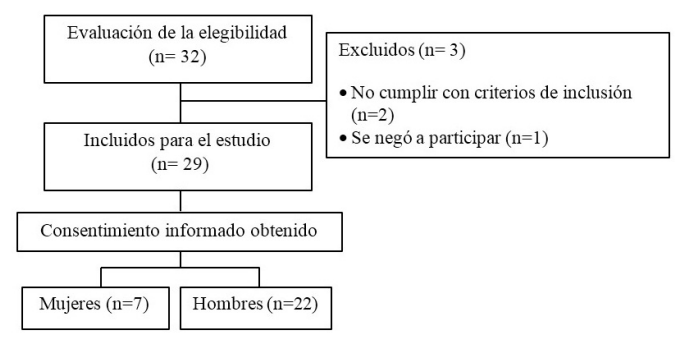

Figura 1. Flujograma Selección de la población. Fuente: Elaboración propia.

el $17.2 \%$ de sexo femenino. El 89.7\% se caracterizó con dominancia diestra. El 82.8\% refiere practicar la religión católica, $48.3 \%$ tiene estudios de nivel superior, el $41.4 \%$ se encuentra pensionado. En la Tabla 1, se especifica la información referente a los datos socio-demográficos y clínicos de la población.

La aplicación del cuestionario ocupacional reflejó un tiempo promedio de horas diarias invertidas en actividades laborales de 2 horas, en actividades de la vida diaria 11 horas, en actividades de recreación 5 horas y en actividades de descanso 6 horas aproximadamente como aparece en la Tabla 2.

$\mathrm{Al}$ interpretar los resultados del listado de roles propuesto por el modelo de ocupación humana, se encuentra que los roles actuales que más desempeña la población son el rol de miembro de familia y amigo con un $93 \%$ cada uno y los que menos desempeñan son el rol de miembro activo de grupo religioso con un $34 \%$ y el rol de estudiante con un 38\% de la población (ver Tabla 3).

Se puede observar que para rol de estudiante el $100 \%$ de la población lo desempeño en el pasado y el 69\% tiene algún interés en desempeñarlo en el futuro. Por otro lado, el rol de trabajador fue desempeñado en el pasado por el $97 \%$ de la población, sin embargo el interés por desempeñarlo en el futuro es del 65\% de la población.

Otro rol explorado y en el cual se encuentran hallazgos interesantes, es el rol de voluntario, en el cual solo el 59\% de la población lo desempeñaba en el pasado pero el $79 \%$ identifica que le gustaría desempeñarlo en el futuro. El rol anterior se complementa con el rol de proveedor de cuidados ya que el $72 \%$ lo desempeńó en el pasado y al $76 \%$ le gustaría desempeñarlo en el futuro.

En el rol referido a la participación como miembro de grupo religioso se evidencia que el porcentaje de personas que desempeñaban este rol disminuyó en el presente en un $10 \%$ y vuelve a aumentar en la percepción hacia el futuro en un $6.9 \%$. 
Tabla 1. Descripción sociodemográfica $(n=29)$ rever dados em comparação a figura anterior.

\begin{tabular}{|c|c|c|c|c|}
\hline \multicolumn{2}{|c|}{ Variable } & Frecuencia & $\%$ & Media/moda \\
\hline \multirow{4}{*}{ Edad (años) } & $40-49$ & 10 & 34.5 & \multirow{4}{*}{53.41} \\
\hline & $50-59$ & 14 & 48.3 & \\
\hline & $60-69$ & 4 & 13.8 & \\
\hline & $70-79$ & 1 & 3.4 & \\
\hline \multirow{2}{*}{ Sexo } & 1. Femenino & 5 & 17.2 & \multirow{2}{*}{2} \\
\hline & 2. Masculino & 24 & 82.8 & \\
\hline \multirow{2}{*}{ Natural } & 1. Bogotá & 9 & 31 & \multirow{2}{*}{2} \\
\hline & 2. Fuera de Bogotá & 20 & 69 & \\
\hline \multirow{2}{*}{ Procedencia } & 1. Bogotá & 26 & 89.7 & \multirow{2}{*}{1} \\
\hline & 2. Fuera de Bogotá & 3 & 10.3 & \\
\hline \multirow{2}{*}{ Dominancia } & 1. Derecha & 26 & 89.7 & \multirow{2}{*}{1} \\
\hline & 2. Izquierda & 3 & 10.3 & \\
\hline \multirow{3}{*}{ Religión } & 1. Católica & 24 & 82.8 & \multirow{3}{*}{1} \\
\hline & 2. Otra & 41 & 3.3 & \\
\hline & 3. Sin religión & 4 & 13.8 & \\
\hline \multirow{3}{*}{ Nivel educativo } & 1. Básica Primaria & 7 & 24.1 & \multirow{3}{*}{3} \\
\hline & 2. Básica Secundaria & 8 & 27.6 & \\
\hline & 3. Superior & 14 & 48.3 & \\
\hline \multirow{4}{*}{ Ocupación } & 1. Trabaja & 8 & 27.6 & \multirow{4}{*}{3} \\
\hline & 2. No Trabaja & 4 & 13.8 & \\
\hline & 3. Pensionado & 12 & 41.4 & \\
\hline & 4. Trabaja/Pensionado & 5 & 17.2 & \\
\hline
\end{tabular}

Fuente: Elaboración propia.

Tabla 2. Promedio de horas diarias invertidas en actividades ocupacionales $(\mathrm{n}=29)$.

\begin{tabular}{lrl}
\hline \multicolumn{1}{c}{$\begin{array}{c}\text { Actividades } \\
\text { ocupacionales/horas } \\
\text { diarias invertidas }\end{array}$} & Media & D.T \\
\hline Trabajo & 2.1 & 3.71 \\
Vida diaria & 10.7 & 4.57 \\
Recreación & 4.7 & 3.52 \\
Descanso & 6.2 & 4.41 \\
D.T: Desviación típica & & \\
\hline
\end{tabular}

Fuente: Elaboración propia.

Finalmente, al explorar la independencia en actividades instrumentales de la vida diaria con el índice de Lawton y Brody, se evidencia que el $65.5 \%$ de la población es independiente como se observa en la Tabla 4.

\section{Discusion}

A lo largo de la vida, las personas con VIH/SIDA, enfrentan una serie de cambios sociales, físicos, cognitivos y emocionales que impactan en todas las áreas ocupacionales: ocio, autocuidado, trabajo y escolaridad (SALZ, 2001).

Las personas que viven con VIH/SIDA experimentan pérdida de independencia física, dolor, problemas de memoria y juicio, ansiedad, depresión, cambios en los roles, baja autoestima, ausencia de la red de apoyo (familia, comunidad); en consecuencia se ve afectado el desempeño ocupacional ya que se ve comprometida la participación de las personas y su vinculación en roles ocupacionales propios de su edad y de su contexto cultural. Sin embargo, en la población participante del proyecto, es evidente que aún se mantienen independientes en el ejercicio de sus actividades instrumentales de la vida diaria y en el desempeño de roles como el de amigo y miembro de familia, lo anterior puede deberse a los criterios de inclusión y exclusión determinados por el grupo investigador, sin embargo el rol laboral se transforma y deja de ocupar un lugar prioritario de este grupo poblacional, de este modo, Gutterman (1990) ha demostrado que existen barreras para acceder al empleo y a otro tipo de participación productiva, además se ha evidenciado que la organización de la rutina cotidiana se modifica, las expectativas sobre el rol productivo (escolar o laboral) disminuyen, la exploración de los propios intereses para el desarrollo se limitan, afectando de esta manera el ejercicio de los roles ocupacionales y la calidad de vida del individuo con VIH/SIDA.

Un déficit en el desempeño de actividades productivas puede conducir a la depresión, a la percepción de pérdida del control, a dificultades 
Tabla 3. Desempeño de Roles ( $\mathrm{n}=29)$.

\begin{tabular}{|c|c|c|c|c|c|c|}
\hline \multirow{2}{*}{ Rol } & \multicolumn{2}{|c|}{ Pasado } & \multicolumn{2}{|c|}{ Presente } & \multicolumn{2}{|c|}{ Futuro } \\
\hline & $\mathrm{f}$ & $\%$ & $\mathbf{F}$ & $\%$ & f & $\%$ \\
\hline \multirow{2}{*}{ Estudiante } & ND: 0 & 0 & ND: 18 & 62.1 & ND: 9 & 31 \\
\hline & D:29 & 100 & D: 11 & 37.9 & D: 20 & 69 \\
\hline \multirow{2}{*}{ Trabajador } & ND: 1 & 3.4 & ND: 13 & 44.8 & ND: 10 & 34.5 \\
\hline & D: 28 & 96.6 & D: 16 & 55.2 & D: 19 & 65.5 \\
\hline \multirow{2}{*}{ Voluntario } & ND: 12 & 41.4 & ND: 15 & 51.7 & ND: 6 & 20.7 \\
\hline & D: 17 & 58.6 & D: 14 & 48.3 & D: 23 & 79.3 \\
\hline \multirow{2}{*}{ Proveedor de cuidados } & ND: 8 & 27.6 & ND: 15 & 51.7 & ND: 7 & 24.1 \\
\hline & D: 21 & 72.4 & D: 14 & 48.3 & D: 22 & 75.9 \\
\hline \multirow{2}{*}{$\begin{array}{l}\text { Ama de casa/ } \\
\text { Dona de casa }\end{array}$} & ND: 7 & 24.1 & ND: 8 & 27.6 & ND: 9 & 31 \\
\hline & D: 22 & 75.9 & D: 21 & 72.4 & D: 20 & 69 \\
\hline \multirow{2}{*}{ Amigo } & ND: 3 & 10.3 & ND: 2 & 6,9 & ND: 2 & 6.9 \\
\hline & D: 26 & 89.7 & D: 27 & 93,1 & D: 27 & 93.1 \\
\hline \multirow{2}{*}{ Miembro de la Familia } & ND: 1 & 3.4 & ND: 2 & 6.9 & ND: 2 & 6.9 \\
\hline & D: 28 & 96.6 & D: 27 & 93.1 & D: 27 & 93.1 \\
\hline \multirow{2}{*}{$\begin{array}{l}\text { Miembro Activo de } \\
\text { Grupo Religioso }\end{array}$} & ND: 16 & 55.2 & ND: 19 & 65.5 & ND: 17 & 58.6 \\
\hline & D: 13 & 44.8 & D: 10 & 34.5 & D: 12 & 41.4 \\
\hline \multirow{2}{*}{ Aficionado/amador } & ND: 6 & 20.7 & ND: 6 & 20.7 & ND: 5 & 17.2 \\
\hline & D: 23 & 79.3 & D: 23 & 79.3 & D: 24 & 82.8 \\
\hline \multirow{2}{*}{$\begin{array}{l}\text { Participante de } \\
\text { Organizaciones }\end{array}$} & ND: 14 & 48.3 & ND: 16 & 55.2 & ND: 11 & 37.9 \\
\hline & D: 15 & 51.7 & D: 13 & 44.8 & D: 18 & 62.1 \\
\hline
\end{tabular}

$1=D$ : Lo desempeña $0=N D$ : No lo desempeña. Fuente: Elaboración propia.

Tabla 4. Índice de Lawton y Brody. Independencia en AIVD ( $\mathrm{n}=29)$.

\begin{tabular}{lcc}
\hline \multicolumn{1}{c}{ Independencia en AIVD } & Frecuencia & $\%$ \\
\hline 1.Independiente & 19 & 65.5 \\
2.Precisa ayuda & 9 & 31 \\
3.Dependiente & 1 & 3.4 \\
\hline
\end{tabular}

Fuente: Elaboración propia.

financieras, estigmas sociales, mal estado de salud y reducción de la independencia (SALZ, 2001).

Dickerson y Oakley citado por Braveman y Kielhofner (2006), en 1993 sugieren que la diferencia más grande e importante en los roles asumidos por esta población ocurren en los roles de estudiante, trabajador y cuidador. Lo cual se evidencia en los resultados descritos anteriormente, ya que existe una disminución actual en el desempeño del rol de trabajador. De acuerdo con Mosey (1996), la actividad laboral "incluye las actividades necesarias para participar en un empleo remunerado o en actividades de voluntariado" citado por American Occupational Therapy Association (2008, p. 632). Algunos estudios muestran (BRAVEMAN; KIELHOFNER, 2006) que obtener y mantener un empleo constituye un obstáculo para personas con VIH, pero su existencia se convierte en un factor importante para reducir el estigma social, promover la socialización, la independencia, el autoestima y la autoidentidad, además de la participación activa en actividades de la vida diaria.

Por otro lado, según la literatura, participar activamente de relaciones familiares, de amistad, amorosas, etc., constituyen un constante e intensivo apoyo emocional donde se satisface la necesidad del cuidado hacía otras personas, lo cual brinda sentido de pertenencia y alianza, es decir el sentirse aceptado y no discriminado por su red apoyo (BEDELL, 2000; BASAVARAJ; NAVYA; RASHMI, 2010). Los hallazgos encontrados en el estudio, muestran que el rol de amigo y miembro de familia se desempeńa de manera homogénea en el transcurrir del tiempo.

Por otro lado es importante mencionar que las actividades de la vida diaria (AVD) incluyen las actividades básicas orientadas al cuidado del propio cuerpo y las actividades instrumentales de la vida diaria que son todas aquellas dirigidas al apoyo a la vida cotidiana en la casa y en la comunidad y que requieren mayor complejidad en sus interacciones que las ejercidas en las actividades de autocuidado (AMERICAN..., 2008). De acuerdo con esta explicación, la población participante reporta ser independiente en su mayoría, sin embargo existe un porcentaje mínimo que requiere atención en este aspecto como lo reporta la literatura.

Es de resaltar que el ejercicio del rol de ama/o de casa se relaciona con el nivel de independencia 
en AVD que tiene la población participante del estudio, puesto que la mayoría de ellos, realizan actividades como preparar alimentos, manejar el dinero, movilizarse en transporte público, comunicarse telefónicamente y manejar los medicamentos. Sin embargo, la evidencia muestra que el sentido de discapacidad experimentado por las personas que presentan esta enfermedad está relacionado con la dificultad en el desempeño independiente de actividades básicas e instrumentales de la vida diaria además de la participación activa en la comunidad (ANANDAN et al., 2006).

A pesar de que la recreación corresponde a aquellas actividades placenteras desarrolladas en el tiempo libre que comprometen un valor cultural el cual se dirige a satisfacer necesidades de descanso, entretenimiento, expresión, aventura y socialización, además de proveer estructura para la vida (STEVENS-RARCHFORD; KRAUSE, 2004), se puede observar que la población del estudio dedica un promedio de 4.7 horas a este tipo de actividades. Por otro lado, la actividad de descanso es desempeñada por los participantes en un promedio de 6.2 horas diarias. La Asociación Americana de Terapia Ocupacional (AMERICAN..., 2008, p. 11) cita a Nurit y Michel quienes afirman que:

El descanso es toda acción silenciosa y sin esfuerzo que interrumpe la actividad física y mental, resultando en un estado relajado. Incluye identificar la necesidad de relajarse; reducir la participación agotadora en actividades físicas, mentales y sociales; participar en la relajación u otros esfuerzos que reestablezcan la energía, calma y un renovado interés en esta participación.

La literatura ha proporcionado información referente al impacto que dicho diagnóstico de VIH tiene a nivel del desempeño ocupacional de un individuo que vive con VIH SIDA. Se destaca que debido a las causas del contagio de VIH, el terapeuta ocupacional debe ayudar al usuario a afrontar y manejar la estigmatización social existente. Como área principal en el adulto, se reconoce que el retorno laboral o el acceso a un puesto de trabajo son directamente afectados a causa de la estigmatización del virus. Además de lo anterior, existen autores que coinciden en la perdida y cambio significativo de roles, debido a la sintomatología, las comorbilidades y los efectos adversos de la medicación.

\section{Conclusión}

Se hace evidente la importancia y pertinencia que tiene para Terapia Ocupacional estudiar el impacto del VIH sobre las diferentes etapas del ciclo vital principalmente en los adultos y los cambios de roles que se asumen con esta condición de salud. Es concluyente a su vez la limitación del estudio respecto al número de participantes en el proyecto y al criterio de inclusión referido a la edad, ya que esto sumado a la posibilidad de acceder a un tratamiento integral y estar vinculado a un programa de atención integral, resulta para estos pacientes, en gran medida la posibilidad de mantenerse independientes en sus actividades diarias. Vale la pena mencionar que los resultados del estudio no son generalizables a la población con VIH ya que habría que considerar variables como la etapa de la enfermedad, el contexto en el que viven y las comorbilidades.

Cuando una persona debe asumir nuevos roles debido a cambios significativos por el inicio de una nueva etapa del ciclo vital o el curso del VIH, se cuestiona cómo dichos cambios influyen en la vida social y laboral, en la realización de las actividades de la vida diaria del paciente y por tanto en el desempeño ocupacional.

Es fundamental el papel del terapeuta ocupacional en los programas de manejo integral de las personas que viven con VIH/SIDA con el fin de brindar intervenciones que promuevan comportamientos adaptativos frente a los cambios en función de los roles, para impactar en el desempeńo ocupacional (SALZ, 2001).

Debido al alto interés de desempeñar el rol de voluntario y de proveedor de cuidados en el futuro, surge la necesidad de crear estrategias que fomenten el trabajo colaborativo y de ocio productivo para ser consideradas como opción ocupacional para este grupo poblacional.

Las funciones de productividad se componen de rutinas y tareas que permiten a una persona desempeñarse exitosamente en las distintas áreas de ocupación (Actividades básicas de la vida diaria, actividades instrumentales de la vida diaria, descanso, sueño, educación, trabajo, juego, ocio/tiempo libre y participación social) las cuales se ven afectadas por los cambios ligados a curso de la enfermedad del VIH/SIDA. La intervención desde Terapia Ocupacional muestra resultados significativos respecto al beneficio para los pacientes que viven con VIH/SIDA, ya que la intervención promueve la calidad de vida reduciendo el sentimiento de soledad y aumentando el de bienestar, también fortalece y entrena las habilidades para desempeñarse en actividades de la vida diaria y de esta manera disminuye el tiempo de institucionalización de estos pacientes (BRAVEMAN; HELFRICH, 2001). 
Mientras se brinda el servicio de Terapia Ocupacional tradicional, el profesional actúa como agente de cambio a través del rol de educador para proveer ayuda en la creación de ambientes que favorezcan cambios en la educación en salud, promoción de las actividades que apoyan física, emocional y espiritualmente, facilitando las respuestas adaptativas, promoviendo la motivación e interviniendo en procesos de mejoramiento del desempeńo ocupacional y promoción de la participación (GUTTERMAN, 1990; RANKA; CHAPPARO, 2010).

\section{Referencias}

AMERICAN OCCUPATIONAL THERAPY ASSOCIATION - AOTA. Occupational therapy practice framework: Domain and process. American Journal Occupational Therapy, Boston, v. 62, n. 6, p. 625-683, 2008.

ANANDAN, N. et al. Impairments and perceived competence in persons livings with HIV/AIDS. Work, Netherlands, v. 27, n. 3, p. 255-266, 2006.

BARKEY, V. et al. Barriers and facilitators to participation in work among Canadian Women living with HIV/ AIDS. Canadian Journal of Occupational Therapy, Ottowa, v. 74, n. 4, p. 269-75, 2009.

BASAVARAJ, K. H.; NAVYA, M. A.; RASHMI, R. Quality of life in HIV/AIDS. Indian Journal Sexually Transmitted Diseases, Madras, v. 31, n. 2, p. 75-80, 2010.

BEDELL, G. Daily life for eight urban gay men with HIVIAIDS. The American Journal of Occupational Therapy, Boston, v. 54, n. 2, p. 197-206, 2000.

BRAGANÇA, M.; PALHA, A. Trastornos neurognitivos asociados con la infección por el VIH. Actas Españolas de Psiquiatría, Spain, v. 39, n. 6, p. 374-383, 2011.

BRAVEMAN, B.; HELFRICH, C. A. Occupational identity: exploring the narratives of three men living with AIDS. Journal of Occupational Science, North Terrace, v. 8, n. 2, p. 25-31, 2001.

BRAVEMAN, B.; KIELHOFNER, G. HIV/AIDS and employment: the continuing challenge. Work, Netherlands, v. 27, n. 3, p. 205-207, 2006.

CENTRAL INTELLIGENCE AGENCY. The world factbook: country comparison: HIV/AIDS - people living with HIV/AIDS. Bogotá. Disponible: <https:// www.cia.gov/library/publications/the-world-factbook/ rankorder/2156rank.html? countryname=Colombia\&co untrycode $=$ co\&regionCode $=$ soa\&rank $=32>$. Access on: 29 abr. 2014.

COLOMBIA. Ministerio de Salud. Resolución $\mathrm{n}^{\circ}$ 008430, de 1993 del 4 de octubre de 1993. Por la cual se establecen las normas científicas, técnicas y adminis- trativas para la investigación en salud. Diário Oficial da Colômbia, Bogotá, 4 oct. 1993. p. 1-12.

COLOMBIA. Ley no 972, de 15 de Julio de 2005. Modificada por el art. 36, Decreto Nacional 126 de 2010, en lo relativo a las multas por la cual se adoptan normas para mejorar la atención por parte del Estado colombiano de la población que padece de enfermedades ruinosas o catastróficas, especialmente el VIH/ Sida. Diário Oficial da Colômbia, Bogotá, 15 julio 2005. Disponible: <http://www.alcaldiabogota.gov.co/sisjur/ normas/Norma1.jsp?i=17080 > Access on: 22 abr. 2014.

COLOMBIA. Ministerio de Salud y Protección Social. Boletín epidemiológico, situación del VIH/SIDA Colombia. Bogotá: Ministerio de Salud y Protección Social, 2014.

GUTTERMAN, L. A day treatment program for persons with AIDS. American Journal of Occupational Therapy, Boston, v. 44, n. 3, p. 234-237, 1990.

JIMÉNEZ, P. E. et al. Evaluation of the instrumental activities of daily living following a stroke by means of the Lawton and Brody scale. Revista de Neurologia, Barcelona, v. 55, n. 6, p. 337-342, 2012.

KIELHOFNER, G. A model of human occupation theory and application. Baltimore: Williams \& Wilkins, 1995.

KIELHOFNER, G. et al. A controlled study of services to enhance productive participation among people with HIV/AIDS. American Journal of Occupational Therapy, Boston, v. 62, n. 1, p. 36-45, 2008.

KRONEMBERG, F.; SIMO, S.; POLLARD, N. Terapia Ocupacional sin fronteras aprendiendo del espiritu de supervivientes. Madrid: E. M. Panamericana, 2007.

MOSEY, A. C. Applied scientific inquiry in the health professions: an epistemological orientation. Bethesda: MD: American Occupational Therapy Association, 1996.

NEHER, R. A.; LEITNER, T. Recombination rate and selection strength in HIV Intra-patient evolution. PLoS Computational Biology, Los Alamos Nuevo México, v. 6, n. 1, p. 1-7, 2010.

OACKLEY, F.; KIELHOFNER, G.; BARRIS, R. Role checklist: development and empírical assessment of reliability. British Journal Occupational Therapy, Monthly, v. 6, n. 3, p. 157-170, 1986.

RANKA, J. L.; CHAPPARO, C. J. Assessment of productivity performance in men with HIV Associated Neurocognitive Disorder (HAND). Work, Netherlands, v. 36, n. 2, p. 193-206, 2010.

RIOPEL, N.; KIELHOFNER, G.; HAWKINGS, J. The relationships between volition, activity pattern, and life satisfaction in the elderly. The American Journal of Occupational Therapy, Boston, v. 40, n. 4, p. 278-283, 1986. 
SALZ, F. HIV/AIDS and work: the implications for occupational therapy. Work, Netherlands, v. 16, n. 3, p. 269-272, 2001.

STEVENS, R.; KRAUSE, A. Visually impaired older adults and home-based leisure activities: the effects of person-environment congruence. Journal of Visual Im- pairment and Blindness, New York, v. 98, n. 1, p. 14-27, 2004.

TEVA, I. et al. Situación epidemiológica actual del VIH/ SIDA en Latinoamérica en la primera década del siglo XXI: análisis de las diferencias entre países. Revista Médica de Chile, Santiago, v. 140, n. 1, p. 50-58, 2012.

\section{Contribución de los Autores}

Todos los autores aportaron en la construcción del texto, desde la búsqueda de evidencia, desarrollo del proyecto, análisis de resultados y redacción del documento. Todos los autores aproban la version final del texto. 